\title{
BMJ Open Estimation of total cardiovascular risk using the 2019 WHO CVD prediction charts and comparison of population- level costs based on alternative drug therapy guidelines: a population-based study of adults in Bangladesh
}

Jessica Yasmine Islam (D) , , ${ }^{1,2}$ M Mostafa Zaman, ${ }^{1}$ Mohammad Moniruzzaman (D) , ${ }^{3}$ Shawkat Ara Shakoor, ${ }^{4}$ A H M Enayet Hossain ${ }^{4}$

To cite: Islam JY, Zaman MM, Moniruzzaman M, et al. Estimation of total cardiovascular risk using the 2019 WHO CVD prediction charts and comparison of populationlevel costs based on alternative drug therapy guidelines: a population-based study of adults in Bangladesh. BMJ Open 2020;10:e035842. doi:10.1136/ bmjopen-2019-035842

- Prepublication history and additional material for this paper are available online. To view these files, please visit the journal online (http://dx.doi. org/10.1136/bmjopen-2019035842).

Received 18 November 2019 Revised 21 April 2020 Accepted 22 April 2020

Check for updates

(C) Author(s) (or their employer(s)) 2020. Re-use permitted under CC BY. Published by BMJ.

For numbered affiliations see end of article.

Correspondence to Dr Jessica Yasmine Islam; islamjy@email.unc.edu

\section{ABSTRACT}

Objective The objective of this study was to estimate the population distribution of 10-year cardiovascular disease (CVD) risk among Bangladeshi adults aged 40 years and above, using the 2019 WHO CVD risk prediction charts. Additionally, we compared the cost of CVD pharmacological treatment based on the total CVD risk (thresholds $\geq 30 \% / \geq 20 \%$ ) and the single risk factor (hypertension) cut-off levels in the Bangladeshi context. Study design Cross-sectional, population-based study. Setting and participants From 2013 to 2014, we collected data from a nationally representative crosssectional survey of adults aged $\geq 40$ years from urban and rural areas of Bangladesh $(n=6189)$. We estimated CVD risk using the 2019 WHO CVD risk prediction charts and categorised as very low $(<5 \%)$, low $(5 \%$ to $<10 \%)$, moderate $(10 \%$ to $<20 \%)$, high $(20 \%$ to $<30 \%)$ and very high risk ( $\geq 30 \%)$. We estimated drug therapy costs using the lowest price of each drug class available (aspirin, thiazide diuretics, statins and ACE inhibitors). We compared the total cost of drug therapy using the total CVD risk versus single risk factor approach.

Primary outcome measures Our primary outcome was 10 -year CVD risk categorised as very low $(<5 \%)$, low $(5 \%$ to $<10 \%)$, moderate $(10 \%$ to $<20 \%)$, high $(20 \%$ to $<30 \%)$ and very high risk ( $\geq 30 \%)$.

Results The majority of adults $(85.2 \%, 95 \% \mathrm{Cl} 84.3$ to 86.1) have a 10-year CVD risk of less than $10 \%$. The proportion of adults with a 10-year CVD risk of $\geq 20 \%$ was $0.51 \%$. Only one adult was categorised with a 10 -year CVD risk of $\geq 30 \%$. Among adults with CVD risk groups of very low, low and moderate, $17.4 \%, 27.9 \%$ and $41.4 \%$ had hypertension (blood pressure (BP) $\geq 140 / 90$ ) and $0.1 \%$, $1.7 \%$ and $2.9 \%$ had severe hypertension ( $\mathrm{BP} \geq 160 / 100$ ), respectively. Using the total CVD risk approach would reduce drug costs per million populations to US\$144540 (risk of $\geq 20 \%$ ).

Conclusion To reduce healthcare expenditure for the prevention and treatment of CVD, a total risk approach using the 2019 WHO CVD risk prediction charts may lead to cost savings.
Strengths and limitations of this study

- Using the recently updated 2019 WHO cardiovascular disease (CVD) risk prediction charts, this study provides evidence for incorporating WHO CVD risk prediction charts into CVD management and healthcare guidelines, and may lead to potential cost savings from a societal perspective.

- The 2019 WHO CVD risk prediction charts should be applied to a population who have not experienced a CVD event in the past; however, we were unable to confirm self-reported medical history of participants using medical charts or health records, leading to the potential for measurement error due to recall bias.

- The cost estimates we present are an underestimate of total costs for CVD-related treatment as the focus of this study is on the cost of pharmacological intervention only as the largest contributor to overall direct costs in Bangladesh.

- Although we present the total number of people estimated to require drug treatment using 2014 population data, we were unable to identify population estimates of only those at risk of their first CVD event due to lack of surveillance data.

- The CVD 10-year risk cut-offs were defined using risk prediction models derived from 85 cohorts mostly from high-income countries, as data from large-scale prospective cohort data from most lowincome and middle-income countries are limited.

\section{BACKGROUND}

Globally, cardiovascular disease (CVD) is the leading cause of death and disproportionately impacts low/middle-income countries (LMICs), where over $75 \%$ of CVD-related deaths occur. ${ }^{1}$ People living in LMICs are at high risk of developing CVDs due to the absence of integrated primary care for early 
detection and prevention of CVD-related risk factors, such as high cholesterol, high blood pressure (BP) and smoking. Limited access to primary care and the growing burden of CVDs are significant causes of poverty in LMICs and hinder the macroeconomic development of many countries. ${ }^{2}$ LMICs are estimated to experience cumulative economic losses exceeding US $\$ 7$ trillion over the next 15 years due to morbidity and mortality caused by non-communicable diseases (NCDs), including CVD. ${ }^{3}$ As such, the significance of the CVD epidemic has gained increasing international recognition over the past decade, leading to the development of several international guidelines for CVD control and prevention. ${ }^{4}$

In 2007, WHO published pocket guidelines, including CVD risk prediction charts, designed for healthcare workers in LMICs to guide patient 10-year risk stratification for heart attack or stroke. ${ }^{5}$ There are two possible strategies suitable for a low-resource setting to assess the risk of a cardiovascular event and identify those at high risk of a fatal CVD outcome: (1) use a single risk factor management strategy, which focuses on one condition at a time, such as hypertension; or, (2) use a more holistic approach considering several risk factors such as age, tobacco use, gender, diabetes diagnosis, body mass index (BMI), BP and blood cholesterol when measured. Through the total risk approach, pocket guidelines help to identify high-risk patients that are in imminent danger of a heart attack or stroke for timely pharmacological treatment or surgical interventions. Additionally, applying the total risk approach via WHO prediction charts in a nationally representative sample provides an opportunity to estimate and monitor the population-level distribution of CVD risk to ultimately inform CVD treatment policy recommendations. ${ }^{67}$

Currently, Bangladesh has not incorporated clinical guidelines for screening or treatment of risk factors based on absolute CVD risk scores and estimation of the population distribution of CVD risk over time. Data are needed to support the implementation of WHO prediction charts as clinical guidelines for Bangladesh, a resource-limited setting, to demonstrate the potential cost savings and benefit of WHO recommendation on CVD prevention. Recently in 2019, WHO updated the CVD risk charts based on newly validated risk prediction models to estimate CVD risk in 21 Global Burden of Disease (GBD) regions. ${ }^{8}$ The newly developed risk prediction models have been calibrated using data from the GBD study to include estimates from LMICs. To our knowledge, here we present the first analysis to apply the updated CVD risk charts among a cohort of Bangladeshi adults. Prior studies conducted in Bangladesh have estimated CVD risk among adults residing in rural areas only and have not included a nationally representative population. ${ }^{9-11}$ Additionally, no prior studies have estimated the potential costs of pharmacological treatment for CVD in Bangladesh using either the single risk factor or total CVD risk approach, as done previously in other settings. ${ }^{7}$ Our objective was to assess the distribution of absolute CVD risk among a nationally representative sample of Bangladeshi adults using the 2019 WHO CVD risk prediction charts recommended for WHO South Asian Region (Bangladesh, Bhutan, India, Nepal and Pakistan). We also compared the costs of drug treatments for CVD prevention using the total cardiovascular risk thresholds at $\geq 20 \%$ and with single risk factor cut-off levels (BP $\geq 140 / 90 \mathrm{~mm} \mathrm{Hg}$ ).

\section{METHODS}

\section{Study design and setting}

Data were analysed from a population-based crosssectional study conducted from September to December 2013 to assess the burden of blindness and low vision among adults in Bangladesh. The target population of this survey included men and women residing in Bangladesh over the age of 40 years. The exclusion criteria included tourists and the institutionalised, such as residents of a military base, hospital, prisons, nursing homes and other such institutions. We provided participants with detailed information regarding the study objectives and procedures using a printed handout prepared in Bengali. Written consent was obtained from participants through signature or, if not possible through thumbprint.

\section{Sampling frame}

We adopted a multistage, geographically clustered, probability-based sampling approach to obtain a nationally representative sample of Bangladesh, as previously described and outlined per WHO STEPwise approach to surveillance (STEPS). ${ }^{12-15}$ Population statistics were obtained using the 2011 national census conducted by the Bangladesh Bureau of Statistics to create the sample frame. ${ }^{16}$ The sampling frame included 64407 primary sampling units (PSUs) covering all 7 divisions of the country. We randomly selected 72 PSUs (25 urban and 47 rural) from 7 divisions, with the probability of selection proportional to the population size of each division. In each PSU, we selected 100 consecutive households as the secondary sampling unit.

For each household, a trained field data collector approached the head of the household or the family member most knowledgeable of the residents to screen for eligible participants. The screening respondent was asked to describe the composition of household residents, which was defined as those who considered the home to be their primary place of residence as of the night before. A list was composed and ordered from the youngest to the oldest age in years starting from 40 years. Using the list of eligible residents, we used the Kish table approach to randomly select one participant from each home. The selected participant was asked to come to a nearby health centre the next day to administer the survey by trained study interviewers and undergo a medical examination by the study physician. Based on the medical review, participants were followed up with by the providers at the health centre for treatment. 


\section{Patient and public involvement}

There was no patient or public involvement in the implementation of this study or interpretation of analytic results.

\section{Data collection}

To ensure effective and uniform data collection, field interviewers underwent a 7-day training on the interview methodology by the study ophthalmologists and epidemiologists. The training included an in-depth review of the survey content and protocol for completing the demographic questionnaire (a modified WHO/PBL Version III). Each member of the data collection team was provided a detailed survey protocol manual outlining the survey activities, the questionnaire interview and information about the duties and responsibilities of all survey personnel.

Demographic data were collected, including age, sex, marital status, educational level and occupation, using a structured questionnaire survey. Data regarding tobacco use, health history and treatment history were also collected. Participants were asked if they smoked (eg, cigarette, hookah, pipe) or if they used smokeless tobacco (eg, chewing tobacco, jorda) to assess the history of tobacco use. Each participant provided medical history for a prior diagnosis of high $\mathrm{BP}$ or hypertension, diabetes, renal disease or any CVDs by a healthcare provider. Medication history was obtained including medication for high $\mathrm{BP}$, diabetes, malaria, steroids, tuberculosis and among women, history of oral contraception. The questionnaire was translated from English to Bengali, adapted, and validated before data collection.

Physical measurements, including height, weight and BP, were collected. Trained field interviewers measured BP using an appropriately calibrated aneroid sphygmomanometer with appropriately sized arm cuffs. BP measurements were consistently taken on the right arm at heart level and elbow assisted while the participant was seated. The initial measurement was performed after $5 \mathrm{~min}$ of rest. After $2 \mathrm{~min}$, the second measurement was taken. The mean of these two BP readings was used as the final BP for each participant. To measure blood glucose levels, we obtained random blood glucose samples. ${ }^{17}$ Capillary blood samples were consistently taken using the right arm and index finger with a glucometer (Accuchek Advantage, Roche Diagnostics Division, Grenzacherstrasse, Switzerland).

\section{Estimation of 10-year CVD risk}

We estimated 10-year CVD risk using the 2019 WHO CVD risk prediction charts. ${ }^{818}$ The prediction charts provide the 10-year risk of a fatal or non-fatal major cardiovascular event, such as myocardial infarction or stroke, based on age, sex, BP, BMI, smoking status, total blood cholesterol and the presence or absence of diabetes mellitus for 14 WHO epidemiological subregions. For each region, two sets of charts have been developed based on the availability of laboratory-based results. As total cholesterol was not measured in our cohort, we used WHO CVD risk non-laboratory-based charts developed for South Asia (including Bangladesh, Bhutan, India, Nepal and Pakistan). The non-laboratory-based risk charts do not account for diabetes diagnosis or total cholesterol levels.

The prediction chart grades CVD risk using the following categories: age (1: 40-44 years; 2 : 45-49 years; 3: 50-54 years; 4: 55-59 years; 5: 60-64 years; 6: 64-69 years; 7: 70-74 years), sex (men and women), smoking (smoker or non-smoker), systolic BP (SBP; $<120 \mathrm{~mm} \mathrm{Hg}$, $120-139,140-159,160$ to $<180$ and $\geq 180)$, and BMI $(<20$, 20-24, 25-29, 30-35 and $\geq 35$ ). The risk categories for 10-year combined acute myocardial infarction and stroke (fatal and non-fatal) are as follows: $<5 \%, 5 \%$ to $<10 \%$, $10 \%$ to $<20 \%, 20 \%$ to $<30 \%$ and $\geq 30 \%$.

Observations with missing values were dropped from the analysis. We did not anticipate any bias from the complete-case analysis approach as the number of missing observations for key variables was less than $2 \%$ : smoking status, $\mathrm{n}=18$ or $0.3 \%$ missing values; $\mathrm{SBP}, \mathrm{n}=22$ or $0.4 \%$ missing values; diastolic $\mathrm{BP}$ (DBP), $\mathrm{n}=25$ or $0.4 \%$ missing values; and $\mathrm{BMI}, \mathrm{n}=30$ or $0.5 \%$,

\section{Data analysis}

We present sociodemographic variables using mean (SD) or the median (IQR) for continuous variables and proportion for categorical variables. We conducted bivariate analyses by sex and age group. We used the $\chi^{2}$ test to assess for any significant differences in CVD risk distribution across sex. For estimating the cost of medicines per million per year (population aged 40 years or older), we used the lowest price of each drug class available in the market (generic preparation of aspirin, thiazide diuretics, statins and ACE inhibitors). Online supplementary appendix table 1 includes further details regarding the specific costs of common drugs used to treat CVD in Bangladesh.

T0 calculate costs, we included the following categories: (1) people with high cardiovascular risk ( $\geq 20 \%$ and $\mathrm{BP} \geq 160 / 100$ ), who are recommended for pharmacological intervention using four different types of drugs for treatment ${ }^{57}$; and (2) people with $\mathrm{BP} \geq 140 / 90$, who are recommended antihypertensive treatment. To calculate the estimated annual total cost of CVD medication treatment per million populations (aged 40 years or older), we multiplied the percentage of the population at risk and the price of medicine in Bangladesh. We included an estimate of the total number of people estimated to require drug treatment as follows: we multiplied the prevalence of the population requiring medication based on each approach by the number of people in the general population in $2013^{19}$ stratified by gender and age group.

\section{RESULTS}

\section{Demographic characteristics}

The mean age of included participants was 52.9 years (men: 53.5 years, women: 52.5 years; table 1 ). The 
Open access

Table 1 Background characteristics of Bangladeshi adult participants $(n=6189)$

\begin{tabular}{|c|c|c|c|c|c|c|}
\hline \multirow[b]{2}{*}{ Characteristics } & \multicolumn{2}{|c|}{ Total $(n=6189)$} & \multicolumn{2}{|c|}{ Men $(n=2824)$} & \multicolumn{2}{|c|}{ Women $(n=3365)$} \\
\hline & Mean (SD) & n (\%) & Mean (SD) & n (\%) & Mean (SD) & n (\%) \\
\hline Age (years) & $52.9(9.9)$ & & $53.5(10.1)$ & & $52.5(9.8)$ & \\
\hline Education (years) & $3.1(4.2)$ & & $4.3(4.8)$ & & 2.1 (3.3) & \\
\hline
\end{tabular}

Area of residence
\begin{tabular}{llrr}
\hline Urban & $1873(30.2)$ & $808(28.6)$ & $1065(31.6)$ \\
\hline Rural & $4316(69.8)$ & $2016(71.4)$ & $2300(68.4)$
\end{tabular}

Occupation

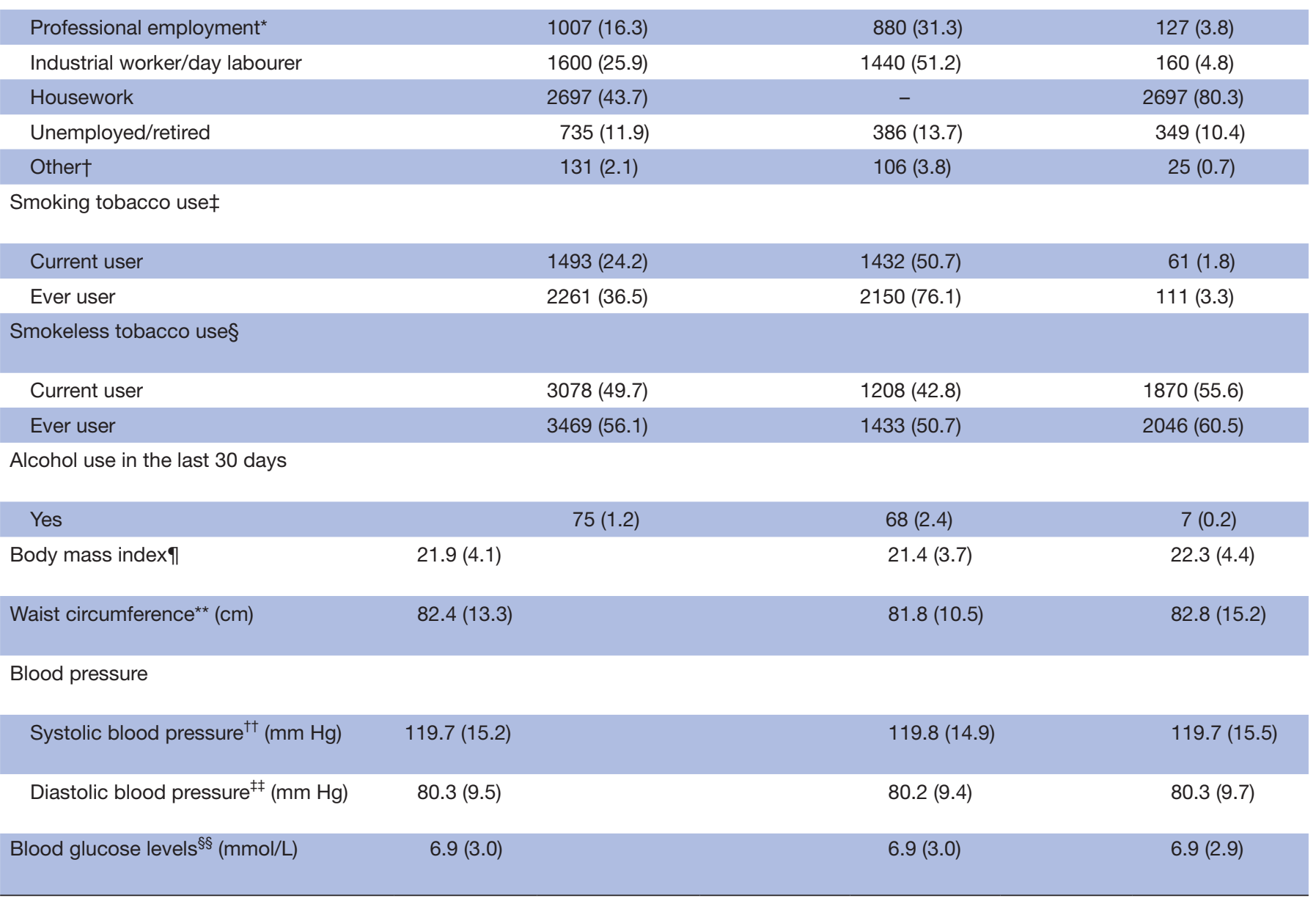

*Professional occupation includes: government employee, private company employee, businessman. tOther occupation includes: shopkeeper, weavers, driver, student, beggar, cook, carpenter and tailor.

fExcluding smokeless tobacco|missing values, $n=18$; men, $n=11$; women, $n=7$.

$\S S$ mokeless tobacco use includes jorda, white leaf (shaada pata), gul and so on.

१Body mass index calculated by weight in kilogram divided by height in metre squared

${ }^{*}$ Missing values, $\mathrm{n}=58$; Male, $\mathrm{n}=25$; Female, $\mathrm{n}=33$

†† Missing values, $n=22$; Male, $n=13$; Female, $n=9$

¥¥Missing values, $n=25$; Male, $n=15$; Female, $n=10$

$\S \S$ Missing values, $n=84$; Male, $n=36$; Female, $n=48$

$\mathrm{CM}$, Centimeters; mmHg, Millimeter of mercury; SD, Standard Deviation.

average level of educational attainment was 3.1 years of education; women were generally less educated than men (2.1 years vs 4.3 years, respectively). The majority $(80 \%)$ of women were housewives, and among men, the most common occupation was an industrial worker or day labourer (51.2\%). Overall, over one-third of participants ever used smoking tobacco, and over half ever used smokeless tobacco. Few participants drank alcohol in the past 30 days $(1.2 \%)$. The mean BMI was $21.9 \mathrm{~kg} / \mathrm{m}^{2}$, and the mean waist circumference was $82.4 \mathrm{~cm}$. 
A
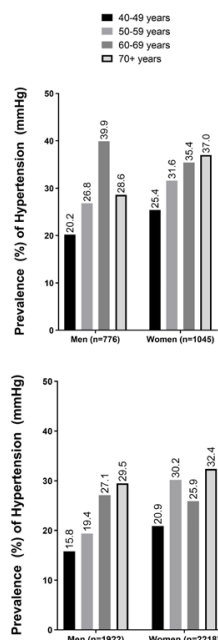
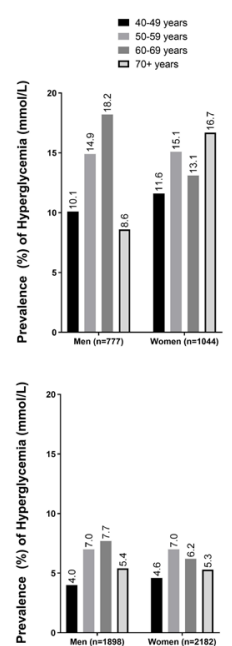
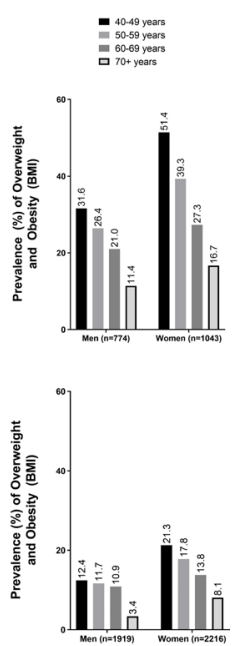

Figure 1 Prevalence of hypertension, hyperglycaemic and overweight and obesity among Bangladeshi adults aged 40 years and above by area of residence and age group. (A) Urban populations. (B) Rural populations. BMI, body mass index.

Overall, the prevalence of hypertension (defined as BP $\geq 140 / 90$ ) increased with age among men and women. Additionally, women had a higher prevalence of hypertension among nearly all age groups in both urban and rural areas. The prevalence of hyperglycaemic (glucose $\geq 11.1 \mathrm{mmol} / \mathrm{L}$ ) was higher among urban adults compared with rural across all age groups. The highest prevalence of hyperglycaemic was observed among urban men aged 60-69 years at $18.2 \%$. Finally, the prevalence of overweight and obesity was higher among urban residents than rural residents. The largest prevalence of overweight and obesity was observed among urban women, with prevalence as high as $51.4 \%$ among women aged $40-49$ years (figure 1).

\section{Distribution of cardiovascular risk}

We summarised the distribution of CVD risk in the population overall and stratified by sex in table 2. Eighty-five per cent of participants had a low $(<10 \%)$ 10-year CVD risk, and this proportion was significantly different across

A

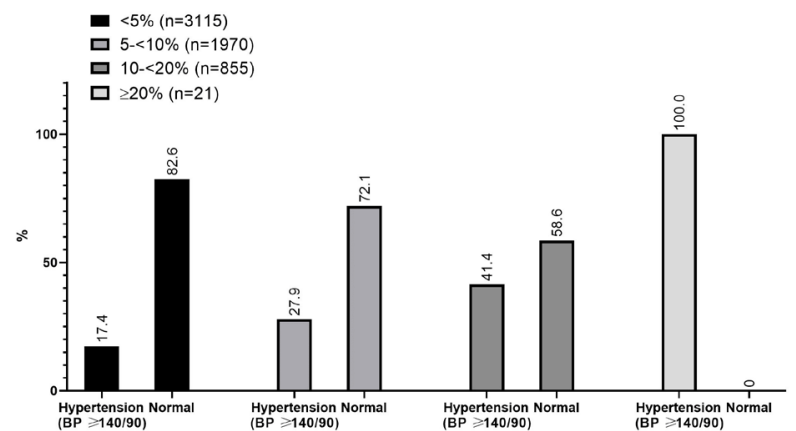

B

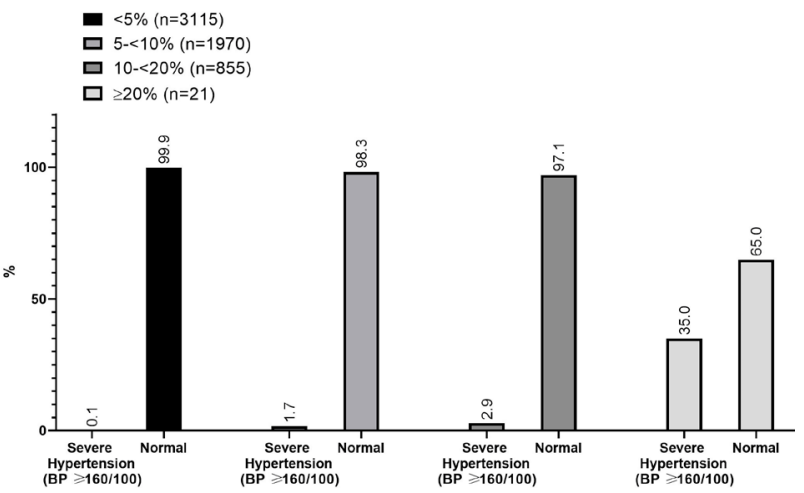

Figure 2 Proportion of adults with hypertension or severe hypertension by cardiovascular disease (CVD) risk group. (A) Per cent of adults with hypertension by CVD risk group. (B) Per cent of adults severe with hypertension by CVD risk group. BP, blood pressure.

sex $(\mathrm{p}<0.001)$. Over half $(63.7 \%)$ of women had a very low $(<5 \%)$ cardiovascular risk. Almost all $(99.5 \%)$ of the study population were categorised as having cardiovascular risk $<20 \%$. A higher proportion of men $(1.0 \%)$ were categorised as high risk than women $(0.1 \% ; \mathrm{p}=<0.001)$. Overall, only one male participant was categorised as very high risk or with a CVD risk of $\geq 30 \%$.

We summarised the prevalence of adults with hypertension by CVD risk group (figure 2). Among those with $10 \%$ to $<20 \%$ CVD risk, we observed a high proportion of hypertension $(41.4 \%)$. In the high-risk group $(\geq 20 \%)$,

Table 2 Ten-year risk of combined myocardial infarction and stroke (fatal and non-fatal) by gender, using the 2019 WHO cardiovascular disease risk non-laboratory-based charts for South Asia $(n=5977)$

\begin{tabular}{|c|c|c|c|c|c|c|c|c|c|c|}
\hline & \multicolumn{3}{|c|}{ Total $(n=5977)$} & \multicolumn{3}{|c|}{ Men $(n=2708)$} & \multicolumn{3}{|c|}{ Women $(n=3269)$} & \multirow[t]{2}{*}{$\begin{array}{l}\text { P } \\
\text { value* }^{*}\end{array}$} \\
\hline & $n$ & $\%$ & $\mathrm{Cl}$ & $n$ & $\%$ & Cl & $\mathbf{n}$ & $\%$ & $\mathrm{Cl}$ & \\
\hline Very low risk $(<5 \%)$ & 3115 & 52.1 & 50.8 to 53.4 & 1034 & 38.2 & 36.6 to 40.0 & 2081 & 63.7 & 62.0 to 65.3 & $<0.001$ \\
\hline Low risk (5\%-10\%) & 1972 & 33.0 & 31.8 to 34.2 & 1047 & 38.7 & 36.8 to 40.5 & 925 & 28.3 & 26.8 to 30.0 & $<0.001$ \\
\hline Moderate risk $(10 \%$ to $<20 \%)$ & 860 & 14.4 & 13.5 to 15.3 & 600 & 22.2 & 20.6 to 23.8 & 260 & 7.8 & 7.0 to 8.9 & $<0.001$ \\
\hline High risk ( $20 \%$ to $<30 \%)$ & 29 & 0.5 & 0.3 to 0.7 & 26 & 1.0 & 0.6 to 1.4 & 3 & 0.1 & 0.0 to 0.2 & $<0.001$ \\
\hline Very high risk ( $\geq 30 \%)$ & 1 & 0.0 & 0.0 to 0.01 & 1 & 0.0 & 0.0 to 0.1 & 0 & 0.0 & 0.0 to 0.0 & 0.272 \\
\hline
\end{tabular}

${ }^{*} \mathrm{P}$ value based on $\chi^{2}$ test. 
Table 3 Estimation of percentage of population requiring drug treatment based on total risk approach in comparison to single risk factor approaches $(n=5977)$

\begin{tabular}{|c|c|c|c|}
\hline & \multicolumn{2}{|c|}{ Cardiovascular (CV) risk $\geq 20 \%$} & Single risk factor approach \\
\hline & $\mathrm{CV}$ risk $\geq 20 \%$ alone, $\%$ & $\begin{array}{l}C V \text { risk } \geq 20 \%+ \\
B P \geq 160 / 100\end{array}$ & $B P \geq 140 / 90$ (SBP $\geq 140+$ isolated raised $D B P), \%$ \\
\hline Women & 0.1 & 1.6 & 26.7 \\
\hline Total & 0.5 & 1.8 & 24.6 \\
\hline
\end{tabular}

BP, blood pressure; DBP, diastolic blood pressure; SBP, systolic blood pressure.

$100 \%$ had hypertension. Additionally, among those with $\geq 20 \%$ CVD risk, we observed that $35 \%$ had severe hypertension $(\mathrm{BP} \geq 160 / 100)$.

\section{Cost of drug treatment}

We were unable to compare the costs of drug treatment at two cardiovascular risk thresholds (30\%-20\%) due to only one male adult with a CVD risk at $\geq 30 \%$. We observed a low proportion of adults with CVD risk $\geq 20 \%$ at $0.5 \%$. When we included $\mathrm{BP} \geq 160 / 100$ measurements, the number of people requiring treatment more than tripled from $0.5 \%$ to $1.8 \%$ (table 3 ). Conversely, if a single risk factor approach was applied, and all those with hypertension (a persistent $\mathrm{SBP} \geq 140$ and/or $\mathrm{DBP} \geq 90$ ) were treated, $24.6 \%$ of the sample would require drug treatment, specifically antihypertensive; more than 20 times the proportion identified when using the total cardiovascular risk approach alone.

\section{Comparison of cost by approach}

Next, we compared the estimated annual cost of medicines per million populations for implementing the total risk approach versus the single risk factor approach. Table 4 shows the estimated number of people aged 40 years or older requiring drug treatment stratified by age group and gender. The estimates showed that if the single risk factor approach is applied in Bangladesh with its percentage of the population at risk and the lowest price of medicine in the country, the cost per million populations (aged 40 years or older) of treating those with $\mathrm{BP} \geq 140 / 90$ would be US\$7111368; if the absolute risk approach were applied, the cost of treating those with a 10 -year risk of CVD $\geq 20 \%$ per million populations (aged 40 years or older) would be US\$144 540, almost 50 times less (figure 3). The cost estimation was based on the percentage of the population at different levels of risk and the differences in the price of generic medicines. For this analysis, we focused on the cost of pharmacological treatment as it is the most critical contributor to the overall direct costs of CVD treatment in Bangladesh. We assumed that other costs of CVD treatment and prevention service delivery, such as health facilities and wages of health workers, are similar for both approaches of service delivery. $^{7}$

\section{DISCUSSION}

Using this nationally representative survey of Bangladeshi adults aged 40 years and above, we found that the majority of adults $(97 \%)$ were at a very low, low or moderate 10 -year risk of myocardial infarction and stroke. The proportion of adults requiring drug treatment rose from $1.0 \%$ to $2.1 \%$ when the threshold for pharmacological intervention was changed from $\geq 20 \%$ alone to $\geq 20 \%$ plus BP of 160/100, respectively, which was lower the proportion than the single risk factor approach (24.6\%). Our data demonstrate that using a single risk factor approach to manage individual cardiovascular risk factors is costlier (US\$7111368 per million population) than using the total risk approach (CVD risk $\geq 20$, US $\$ 144540$ per million population), as a more substantial proportion of adults will need drug treatment. Findings from this analysis support the implementation of clinical guidelines using CVD risk scores calculated using WHO CVD risk prediction charts to appropriately identify patients at the highest risk of CVD development over 10 years in Bangladesh.

In our study using nationally representative data, we found that the 10-year risk of CVD was low $(<10 \%)$ among the vast majority of adults $(85.1 \%)$. Additionally, only $0.5 \%$ of adults were at high risk $(\geq 20 \%)$ of a CVD event within the next 10 years. In this analysis, we applied the 2019 CVD risk charts for South Asia, which are newly developed and now incorporate BMI as part of the prediction chart algorithm. Our results are comparable to regional data presented in the 2019 Lancet publication by WHO CVD Risk Chart Working Group, which showed that $0 \%$ of women from Bhutan, Sri Lanka and Nepal had a CVD risk level above 20\%. Similarly, 0\% of men from Bhutan, and only $<2 \%$ of men from both Sri Lanka and Nepal were categorised with a risk level above $20 \% .^{8}$ These data demonstrate a lower prevalence of CVD risk $\geq 20 \%$ than prior reports from South Asia, which used the original risk prediction charts published in 2007. For example, prior data from $\mathrm{Nepal}^{20}$ and Sri Lanka ${ }^{21}$ showed that $4.3 \%$ and $8.2 \%$ of adults, respectively, were categorised with a high $(\geq 20 \%)$ 10-year risk of a CVD event. Further, analyses from a rural area of South India revealed that $17 \%$ of participants had moderate-to-high risk $(10 \%->20 \%)$ of cardiovascular events per the 2007 


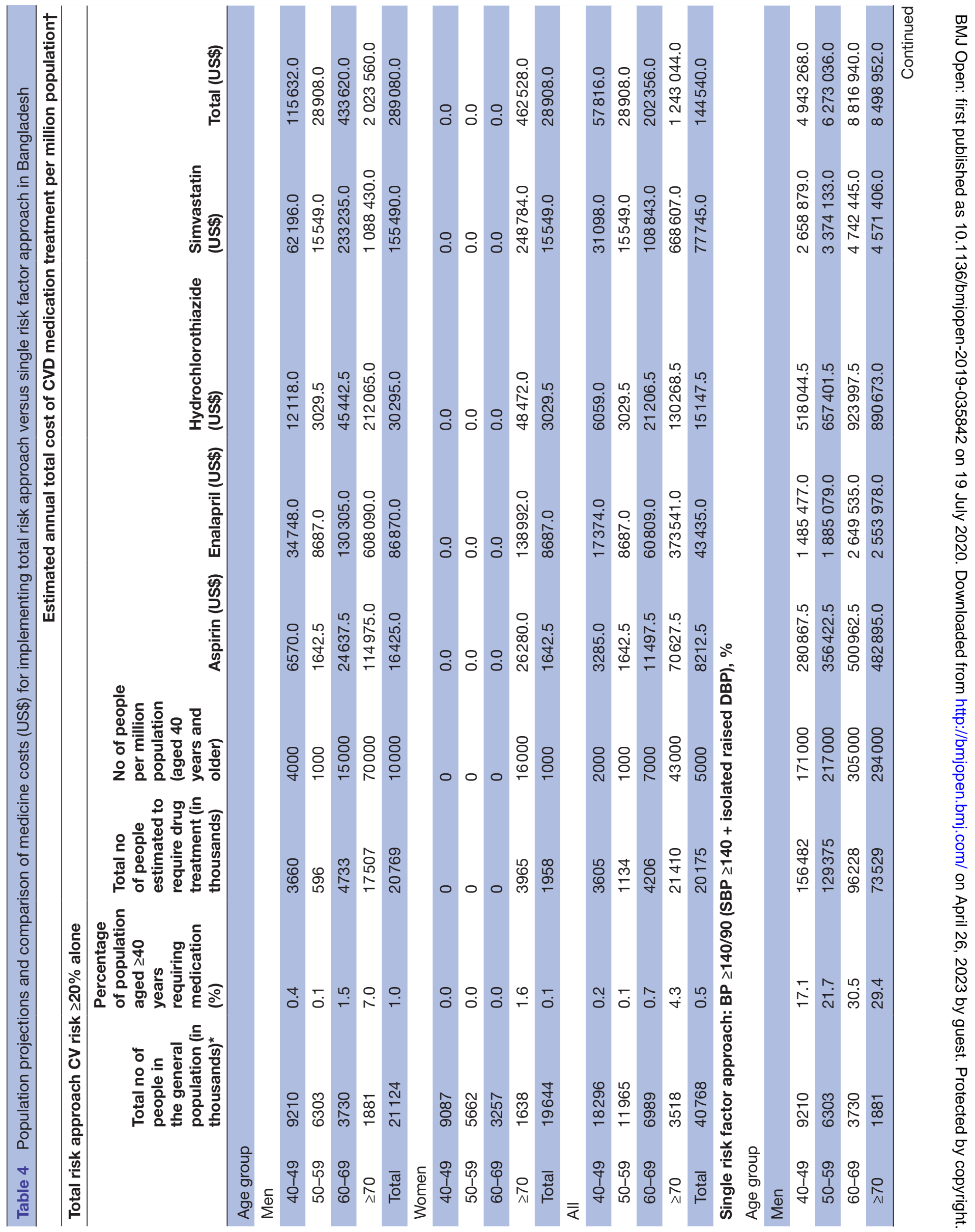




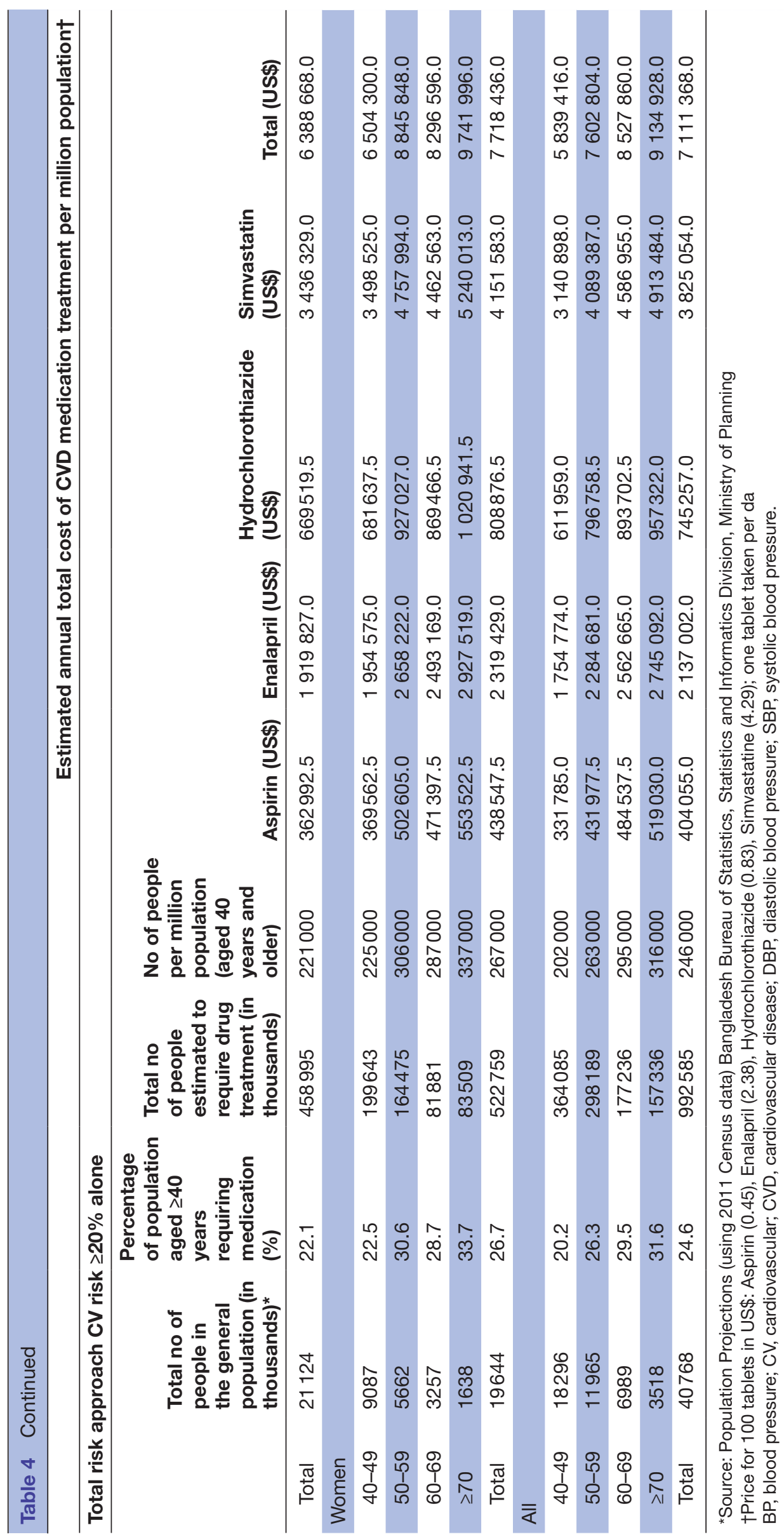




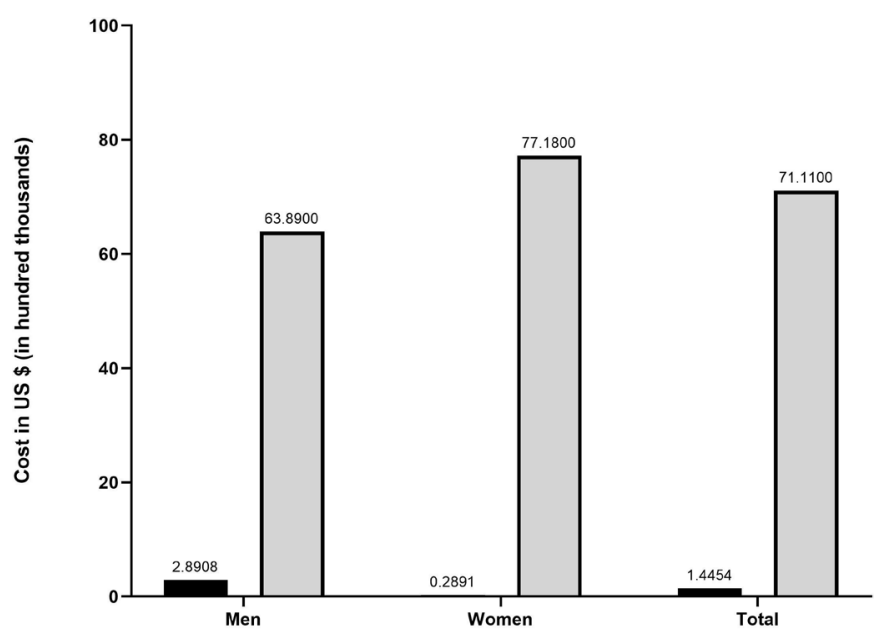

- Estimated annual cost of CVD treatment using the total risk approach $\geqslant 20 \%$ alone

口 Estimated annual cost of CVD treatment using the single risk factor approach

Figure 3 Annual costs of pharmacological treatment for cardiovascular disease (CVD) by sex and risk stratification approach.

WHO prediction charts. ${ }^{22}$ Finally, data collected in 2010 from Pakistan showed that $10 \%$ of adults were categorised with $\geq 20 \%$ CVD risk, with $2.9 \%$ as high as $\geq 40 \%$. When $^{7}$ using the $2019 \mathrm{WHO}$ prediction charts on the population level to measure and monitor trends in total CVD risk in recent years, policy-makers in LMICs should interpret the trends with caution, and assess changes in trends of CVD risk over time using both the 2007 and 2019 WHO risk prediction charts for comparison.

WHO CVD risk charts were developed for use in LMICs and are now more suitable for use in these settings due to the inclusion of data from low-resource regions in the risk prediction model development. While we present the first country-specific analysis using the 2019 risk charts, several prior studies in LMICs have been conducted using the 2007 risk charts. In other countries in Asia, we observe the prevalence of 'high CVD risk' $(\geq 20 \%)$ of $6.0 \%$, $2.3 \%$ and $1.3 \%$ in Mongolia, Malaysia and Cambodia, respectively. ${ }^{23}$ Mendis et al reported the 10-year CVD risk of seven countries and the majority of these countries reported low CVD risk among its adult populations (China $(96.1 \%)$ and Sri Lanka (94.9\%), (Iran (93.9\%), Cuba $(89.7 \%)$, Nigeria $(86.0 \%)$, Georgia $(83.1 \%)$ and Pakistan $(79.2 \%)) .^{7}$ Studies conducted in urban areas of LMICs show varying prevalence of high CVD risk $(\geq 20 \%)$ : for example, one study from Malaysia shows $20.5 \%$ of adults were high risk of CVD,${ }^{24}$ whereas studies from urban Kenya ${ }^{25}$ and Sri Lanka ${ }^{21}$ report less than $10 \%$ of their population had high CVD risk. Specifically in Bangladesh, using the 2007 prediction charts, three prior studies have reported absolute CVD risk among the adult population. ${ }^{1026} 27$ Fatema et al found that $10 \%$ of rural Bangladeshi adults were at high risk $(\geq 20 \%)$ of a CVD event within the next 10 years, and half of these adults fell in the very high-risk category $(\geq 30 \%)$. In another rural
Bangladeshi population, the proportion of participants at a high risk $(\geq 20 \%)$ of a CVD event was $2.1 \% .{ }^{26}$ Finally, in an urban Bangladeshi population of 150 adults, $3.4 \%$ had high CVD risk $(\geq 20 \%)$, which is lower than expected as the population was urban. ${ }^{27}$ No other studies in Bangladesh have been conducted to assess the 10-year risk of CVD using WHO CVD risk prediction charts. We present novel data using the 2019 charts and a nationally representative sample, which may be generalisable to the population of Bangladesh. Data we present may be used to inform policy-makers decisions on clinical guidelines and resource allocation for treatment of CVDs in Bangladesh.

Similar to prior analyses conducted using data from eight LMICs, ${ }^{7}$ our results demonstrate that in the Bangladeshi context using a single risk factor approach to evaluate the risk of CVD-related mortality would cost more than implementing the total risk approach due to higher drug costs. In Bangladesh, about $60 \%$ of out-of-pocket costs patients face go towards drugs directly bought from pharmacies, diagnostics and informal providers. ${ }^{28}$ Currently, Bangladesh does not offer universal health coverage or affordable health insurance plans. The cost of treatment for CVD frequently leads to catastrophic health expenditure and impoverishment; the proportion of catastrophic spending for treatment is highest among those from the lowest quintiles of wealth (14\%) compared with those with high wealth and high socioeconomic status $(6.6 \%) .^{29}$ As such, implementing WHO CVD risk prediction charts may be beneficial to patients in Bangladesh as only those at the highest risk of future CVD would be recommended for treatment.

In addition to benefits to the patient, the total CVD risk approach would also be beneficial to Bangladesh's healthcare system by improving NCD preventive service delivery and the use of guidelines for adequate care. Currently, Bangladesh is categorised by the World Bank as a lower middle-income country with emerging health challenges as the burden of NCDs continues to grow. In 2015, an estimated $67 \%$ of all deaths in Bangladesh were due to NCDs and the risk of premature death from chronic disease among adults aged $30-70$ years was $22 \% .{ }^{30}$ Indeed, CVDs and circulatory diseases are the leading causes of mortality and morbidity in Bangladesh. Despite this substantial burden, preventive services for CVDs in Bangladesh are limited. In 2014, an estimated $16 \%$ of healthcare facilities across the country (ie, hospitals, community clinics) had the resources to diagnose, prescribe treatment for and manage patients with CVDs. ${ }^{31}$ District hospitals (95\%), Upazila health complexes $(81 \%)$ and private hospitals $(77 \%)$ were more likely to provide services for CVDs than other facilities. Only $10 \%$ of community clinics and maternal and child welfare centres, and $17 \%$ of union level facilities, which are the most accessible providers in rural areas, provided any cardiovascular services, and the services at these facilities were limited to the measurement of BP or referrals. ${ }^{31}$ Among facilities with the capacity to offer services for CVD management, about only $20 \%$ used established guidelines for hypertension treatment 
and less than one-third had essential CVD medicines readily available on-site for patients. ${ }^{31}$ By integrating WHO risk prediction charts into the national guidelines for management of hypertension and CVD prevention in Bangladesh, the proportion of facilities using established guidelines may increase as the charts are easy to implement, interpret and access. Additionally, since only onethird of facilities have essential CVD medicines readily available, distributing pharmacological treatment to those at highest risk of premature mortality due to CVD will be crucial.

Although the implementation of a total risk approach may lead to cost savings, there are limitations to implementing the 2019 CVD risk prediction charts. When compared with the single risk factor approach, WHO charts categorise fewer individuals as high risk and may delay the receipt of necessary life-saving treatment. For example, the prediction charts may underestimate CVD risk in certain categories of people such as those with persistent raised $\mathrm{BP} \geq 160 / 100 \mathrm{~mm} \mathrm{Hg}$, blood cholesterol $\geq 8 \mathrm{mmol} / \mathrm{L}$, or those suffering from diabetes with renal disease. ${ }^{5}$ Patients who may fall in these categories should be recommended for intensive lifestyle interventions and appropriate drug therapy; however, the CVD prediction charts will erroneously deny treatment to these potentially high-risk adults. In fact, the risk models used to develop the 2019 CVD risk charts may have underestimated CVD risk due to limitations in the population data used to estimate incidences: Data used to develop the predictions models likely included people already on CVD prevention therapies, such as statins, which have led to an underestimate in CVD risk. ${ }^{8}$ In our study, we underscore the potential for underestimation of CVD risk by comparing the proportion of adults categorised as high risk ( $\geq 20 \%$ CVD risk) to those who would be diagnosed with hypertension ( $\mathrm{BP} \geq 140 / 60)$ and severe hypertension ( $\mathrm{BP} \geq 160 / 100)$. Additionally, we provided a graphical summary of common risk factors of CVD, including hypertension, hyperglycaemia, and overweight and obesity. Despite our very low proportion of adults who would be recommended for treatment based on the risk prediction charts, we observed a high prevalence of these risk factors particularly in urban populations.

Limitations of this analytical approach should be considered when interpreting our results. The CVD 10-year risk cut-offs were defined using risk prediction models derived from 85 cohorts mostly from high-income countries, as data from large-scale prospective cohort data from most LMICs are unavailable. Data were used from the GBD project to recalibrate the models to be representative of LMICs; however, the GBD data do not have country-specific disease risk estimates. As such, the estimation used from each region's chart will most likely apply to the largest country within each region, or from the country where most of the data originated. The risk prediction charts provide approximate estimates of CVD risk in people who do not have established coronary heart disease, stroke or other atherosclerotic diseases. Although we included simvastatin in our pharmacological cost analysis, we were unable to measure total cholesterol or confirm the medical history of participants using medical charts and relied on self-report, leading to the potential for measurement error and recall bias. Additionally, we were unable to categorise participants as diabetic as we did not obtain fasting blood glucose and were only able to categorise adults as hyperglycaemic in our descriptive analysis as we measured random blood glucose. Further, our data were collected in 2013 and may be outdated as population growth in older age groups has been observed in recent years. Our analyses should be replicated using more recent data and future research studies should include the measurement of total cholesterol. Finally, our cost estimates were based on the prevalence of each risk approach in our study sample. Although we present the total number of people estimated to require drug treatment using 2013 population data, estimates of only those at risk of their first CVD event were unavailable due to lack of surveillance data. Nevertheless, our data are valuable as the first analysis to apply the 2019 WHO CVD risk prediction charts to a cohort of adults in Bangladesh. Additionally, we provide data on the comparative cost difference of each approach to underscore the potential cost savings in implementing the total risk approach in Bangladesh. Cost data presented in this analysis may be used in future cost-effectiveness analyses to compare the total risk and single risk factor approach when considering all costs from a societal perspective to inform health policy in Bangladesh.

WHO has outlined global targets in the Global Monitoring Framework for the control of NCDs in LMICs, which prioritises an $80 \%$ of availability of affordable basic technologies and essential medicines necessary to treat significant NCDs, including CVDs in both rural and urban areas of the country. Limited CVD treatment access and weak healthcare infrastructure in Bangladesh are a significant public health concern. As public financing for healthcare is limited in Bangladesh $(\sim 1 \%$ of gross domestic product), public health policies on CVD drug treatment guidelines based on cost estimates, such as out-of-pocket costs, are necessary for effective CVD control. Effective policies should address the potential for overtreatment, which comes at a high cost to both the healthcare system and the patient. The high percentage of the Bangladeshi adult population at low 10-year CVD risk $(<10 \%)$ highlights the potential for reduction of CVD risk through population-wide public health policy and availability of accessible preventive services. However, caution should be taken to ensure that risk stratification approaches are not used in inappropriate clinical circumstances, such as adults with highly uncontrolled hypertension with BP measurements at $160 / 100 \mathrm{~mm} \mathrm{Hg}$.

\section{CONCLUSION}

Our data show that the implementation of a total risk approach compared with a single risk factor approach 
will reduce the healthcare expenditure by lowering drug costs, which accounts for $60 \%$ of out-of-pocket spending in Bangladesh. This approach would be particularly beneficial in Bangladesh, a low-resource country that should prioritise the development of health policy for effective resource allocation in the public health sector. Using the total risk approach would increase service coverage and allow for the distribution of resources to target those at highest risk of experiencing a heart attack or stroke. As the majority of the Bangladeshi adult population aged $\geq 40$ years have a low 10 -year risk of CVD, strategies that target those at highest risk of CVD coupled with public health policies to reduce the population-level risk of CVD may be effective.

\section{Author affiliations}

${ }^{1}$ Non-Communicable Disease Program, World Health Organization Country Office for Bangladesh, Dhaka, Bangladesh

${ }^{2}$ Department of Epidemiology, Gillings School of Global Public Health, University of North Carolina at Chapel Hill, Chapel Hill, North Carolina, USA

${ }^{3}$ Department of Public Health, Shiga University of Medical Science, Otsu, Japan

${ }^{4}$ National Institute of Opthalmology, Dhaka, Bangladesh

Twitter Jessica Yasmine Islam @jyislam and Mohammad Moniruzzaman @ cardiovascular disease

Acknowledgements The study was completed by the National Institute of Ophthalmology (NIO) of Bangladesh with technical assistance from WHO Country Office for Bangladesh. The authors are grateful to the management and field team of NIO consisting of ophthalmologists, other doctors, nurses, technologists and enumerators for their hard works.

Contributors JYI: conceptualised the manuscript, analysed data, interpreted results critically and drafted the manuscript. MMZ: conceptualised the manuscript, designed the study, interpreted results critically, guided manuscript writing and critically reviewed it. MM, SAS and AHMEH: prepared the survey protocol, trained the field team, implemented the survey, processed the data and reviewed the manuscript. SAS is the guarantor of data.

Funding Financial assistance for this study was provided by the WHO Country Office for Bangladesh (WHO Reference: 2013/355662-0, Purchase Order: 200843353, Reg. File: BAN-2013-B7-TSA-0001)

Competing interests None declared.

Patient and public involvement Patients and/or the public were not involved in the design, or conduct, or reporting, or dissemination plans of this research.

Patient consent for publication Not required.

Ethics approval The authors obtained ethical approval for this study from the Institutional Review Board of the National Institute of Ophthalmology.

Provenance and peer review Not commissioned; externally peer reviewed.

Data availability statement Data are available upon reasonable request. The deidentified participant data used and/or analysed during the current study are available from the corresponding author on reasonable request. Please contact M. Mostafa Zaman at zamanm@who.int for further information and guidelines.

Open access This is an open access article distributed in accordance with the Creative Commons Attribution 4.0 Unported (CC BY 4.0) license, which permits others to copy, redistribute, remix, transform and build upon this work for any purpose, provided the original work is properly cited, a link to the licence is given, and indication of whether changes were made. See: https://creativecommons.org/ licenses/by/4.0/.

\section{ORCID iDs}

Jessica Yasmine Islam http://orcid.org/0000-0002-3690-3848

Mohammad Moniruzzaman http://orcid.org/0000-0003-2144-7111

\section{REFERENCES}

1 Checkley W, Ghannem H, Irazola V, et al. Management of ncd in lowand middle-income countries. Glob Heart 2014;9:431-43.

2 Islam SMS, Purnat TD, Phuong NTA, et al. Non-Communicable diseases (NCDS) in developing countries: a symposium report. Global Health 2014;10:81.

3 World Health Organization. Global action plan for the prevention and control of noncommunicable diseases 2013-2020. Geneva, Switzerland: World Health Organization, 2013. http://apps.who.int/ iris/bitstream/10665/94384/1/9789241506236_eng.pdf?ua=1

4 World Health Organization. NCD global monitoring framework, 2017. Available: http://www.who.int/nmh/global_monitoring_framework/en/

5 World Health Organization. Prevention of cardiovascular disease: pocket guidelines for assessment and management of cardiovascular disease. Geneva: World Health Organization, 2007.

6 Ndindjock R, Gedeon J, Mendis S, et al. Potential impact of single-risk-factor versus total risk management for the prevention of cardiovascular events in Seychelles. Bull World Health Organ 2011;89:286-95.

7 Mendis S, Lindholm LH, Anderson SG, et al. Total cardiovascular risk approach to improve efficiency of cardiovascular prevention in resource constrain settings. J Clin Epidemiol 2011;64:1451-62.

8 WHO CVD Risk Chart Working Group. World Health organization cardiovascular disease risk charts: revised models to estimate risk in 21 global regions. Lancet Glob Health 2019;7:e1332-45.

9 Fatema K, Zwar NA, Milton AH, et al. Cardiovascular risk assessment among rural population: findings from a cohort study in a peripheral region of Bangladesh. Public Health 2016;137:73-80.

10 Fatema K, Zwar NA, Milton AH, et al. Application of two versions of the WHO/international Society of hypertension absolute cardiovascular risk assessment tools in a rural Bangladeshi population. BMJ Open 2015;5:e008140.

11 Fatema K, Rahman B, Zwar NA, et al. Short-Term predictive ability of selected cardiovascular risk prediction models in a rural Bangladeshi population: a case-cohort study. BMC Cardiovasc Disord 2016;16:105.

12 Zaman MM, Rahman MM, Rahman MR, et al. Prevalence of risk factors for non-communicable diseases in Bangladesh: results from steps survey 2010. Indian J Public Health 2016;60:17-25.

13 Rahman M, Zaman MM, Islam JY, et al. Prevalence, treatment patterns, and risk factors of hypertension and pre-hypertension among Bangladeshi adults. J Hum Hypertens 2018;32:334-48.

14 Karim MN, Zaman MM, Rahman MM, et al. Sociodemographic determinants of low fruit and vegetable consumption among Bangladeshi adults: results from WHO-STEPS survey 2010. Asia Pac J Public Health 2017;29:189-98.

15 World Health Organization. Surveillance of risk factors for noncommunicable disease: the who stepwise approach. Geneva: World Health Organization, 2001.

16 Bangladesh Bureau of Statistics. Population and housing census 2001, 2009. Available: http://microdata.worldbank.org/index.php/ catalog/1616.

17 World Health Organization. Global report on diabetes. Geneva, Switzerland: World Health Organization, 2016. https://www.who.int/ diabetes/global-report/en/

18 Mendis S, Lindholm LH, Mancia G, et al. World Health organization (who) and International Society of hypertension (ISH) risk prediction charts: assessment of cardiovascular risk for prevention and control of cardiovascular disease in low and middle-income countries. $J$ Hypertens 2007;25:1578-82.

19 Bangladesh Bureau of Statistics. Population projection of Bangladesh dynamics and trends 2011-2061, 2015. Available: http://203.112.218.65:8008/WebTestApplication/userfiles/Image/ PopMonographs/PopulationProjection.pdf.

20 Khanal MK, Ahmed MSAM, Moniruzzaman M, et al. Total cardiovascular risk for next 10 years among rural population of Nepal using WHO/ISH risk prediction chart. BMC Res Notes 2017;10:120.

21 Ranawaka UK, Wijekoon CN, Pathmeswaran A, et al. Risk estimates of cardiovascular diseases in a Sri Lankan community. Ceylon Med J 2016;61:11-17.

22 Ghorpade AG, Shrivastava SR, Kar SS, et al. Estimation of the cardiovascular risk using World health Organization/International Society of hypertension (WHO/ISH) risk prediction charts in a rural population of South India. Int J Health Policy Manag 2015;4:531-6.

23 Otgontuya D, Oum S, Buckley BS, et al. Assessment of total cardiovascular risk using WHO/ISH risk prediction charts in three low and middle income countries in Asia. BMC Public Health 2013;13:539.

24 Su TT, Amiri M, Mohd Hairi F, et al. Prediction of cardiovascular disease risk among low-income urban dwellers in metropolitan Kuala Lumpur, Malaysia. Biomed Res Int 2015;2015:516984. 
25 Vusirikala A, Wekesah F, Kyobutungi C, et al. Assessment of cardiovascular risk in a slum population in Kenya: use of World Health Organisation/International Society of Hypertension (WHO/ISH) risk prediction charts - secondary analyses of a household survey. BMJ Open 2019;9:e029304.

26 Cravedi P, Sharma SK, Bravo RF, et al. Preventing renal and cardiovascular risk by renal function assessment: insights from a cross-sectional study in low-income countries and the USA. BMJ Open 2012;2. doi:10.1136/bmjopen-2012-001357

27 Ahmed MSAM, Moniruzzaman M, Chowdhury S, et al. Cardiovascular risk assessment among urban population of Bangladesh using WHO/ISH risk prediction chart. Int J Epidemiol 2015;44:i202-i.

28 Ministry of Health and Family Welfare, Government of the People's Republic Bangladesh. Multisectoral action plan for the noncommunicable disease control and prevention (2016-2021),
2015. Available: http://www.searo.who.int/entity/ncd_tobacco surveillance/monitoring_fw/pages_from_ban_ncd_action_plan_2016_ 2021_me_not_approved.pdf

29 Bangladesh Bureau of Statistics, Ministry of Planning. Health and morbidity status Survey-2012, 2013. Available: http://bbs.portal.gov. bd/sites/default/files/files/bbs.portal.gov.bd/page/4c7eb0f0_e780_ 4686_b546_b4fa0a8889a5/HMSS.pdf.

30 World Health Organization. Noncommunicable diseases progress monitor 2017, 2017. Available: https://www.who.int/nmh/ publications/ncd-progress-monitor-2017/en/.

31 NIPORT. Bangladesh health facility survey 2014 Dhaka, Bangladesh: National Institute of population research and training (NIPORT), associates for community and population research (ACPR), and ICF international, 2016. Available: https://dhsprogram.com/pubs/pdf/ SPA23/SPA23.pdf 